\title{
A case series of ischemic stroke with coronavirus disease 2019 in two Egyptian centers
}

Nevine El Nahas, Tamer Roushdy, Eman Hamid, Sherien Farag, Hossam Shokri, Mai Fathy (D), Eman Abushady, Ahmed Hazzou and Hany Aref

\begin{abstract}
Background: Severe acute respiratory syndrome coronavirus 2 (SARS-CoV-2) is a novel virus that has been reported to have various neurological manifestations. Cerebrovascular disorders have been encountered as a coronavirus disease 2019 (COVID-19) presentation in our center during the pandemic.

Case presentation: We are presenting 10 cases with cerebrovascular manifestations after having COVID-19 few days prior to stroke.

Conclusion: Cerebrovascular manifestations can occur in association with COVID-19 and may have significant implications on prognosis and management.
\end{abstract}

Keywords: Stroke, Egypt, COVID-19, SARS-CoV-2

\section{Background}

COVID-19 has been declared by the WHO as a pandemic in March 2020. Since then, here have been reports of patients with COVID-19 presenting solely with neurological manifestations [1] or in addition to the classic respiratory symptoms [2]. In a recent study, $36.4 \%$ of patients had neurological manifestations, such as headache, dizziness, acute cerebrovascular diseases, and impaired consciousness [3].

The first COVID-19 case announced in Egypt was on 15 February. At this point, a modified pathway for dealing with suspected cases presenting to ER with stroke and signs of viremia (fever, malaise, and cough) was implemented, in which a CT brain with or without CT angiography according to clinical suspicion of proximal occlusion and CT chest are ordered beside labs and nasopharyngeal swab. Patient is then managed according to guidelines for acute stroke arriving in window of thrombolysis and/or thrombectomy and is admitted to

\footnotetext{
*Correspondence: maifathy23@gmail.com

Neurology Department, Faculty of Medicine, Ain Shams University, Cairo, Egypt
}

(C) The Author(s). 2021 Open Access This article is licensed under a Creative Commons Attribution 4.0 International License, which permits use, sharing, adaptation, distribution and reproduction in any medium or format, as long as you give appropriate credit to the original author(s) and the source, provide a link to the Creative Commons licence, and indicate if changes were made. The images or other third party material in this article are included in the article's Creative Commons licence, unless indicated otherwise in a credit line to the material. If material is not included in the article's Creative Commons licence and your intended use is not permitted by statutory regulation or exceeds the permitted use, you will need to obtain permission directly from the copyright holder. To view a copy of this licence, visit http://creativecommons.org/licenses/by/4.0/. isolation unit, and on obtaining PCR swab results (approximately within $48 \mathrm{~h}$ ), the patient is to be managed at the stroke center or transferred to quarantine hospital with initial stroke management recommendations [4]. In our stroke center, we started seeing COVID patients presenting with acute ischemic stroke (AIS) on 28 April.

In this case series, we are presenting 10 cases with AIS after having COVID-19 few days prior to stroke. They were admitted to Ain Shams University Hospital Stroke Centers. COVID-19 symptoms ranged from fever to dry cough, shortness of breath, and myalgia. All patients were proven to be COVID-19 positive by polymerase chain reaction (PCR) (Table 1).

\section{Cases presentation}

Patient 1

A 65-year-old male, ex-smoker, hypertensive, with ischemic heart disease (IHD), atrial fibrillation (AF), and bronchial asthma since childhood, presented 2 days after COVID-19 symptoms. AIS onset-to-door time (ODT) was 1 and a half hour of right hemiparesis and global aphasia (National Institutes of Health Stroke Scale \\ 望 Springer Open}




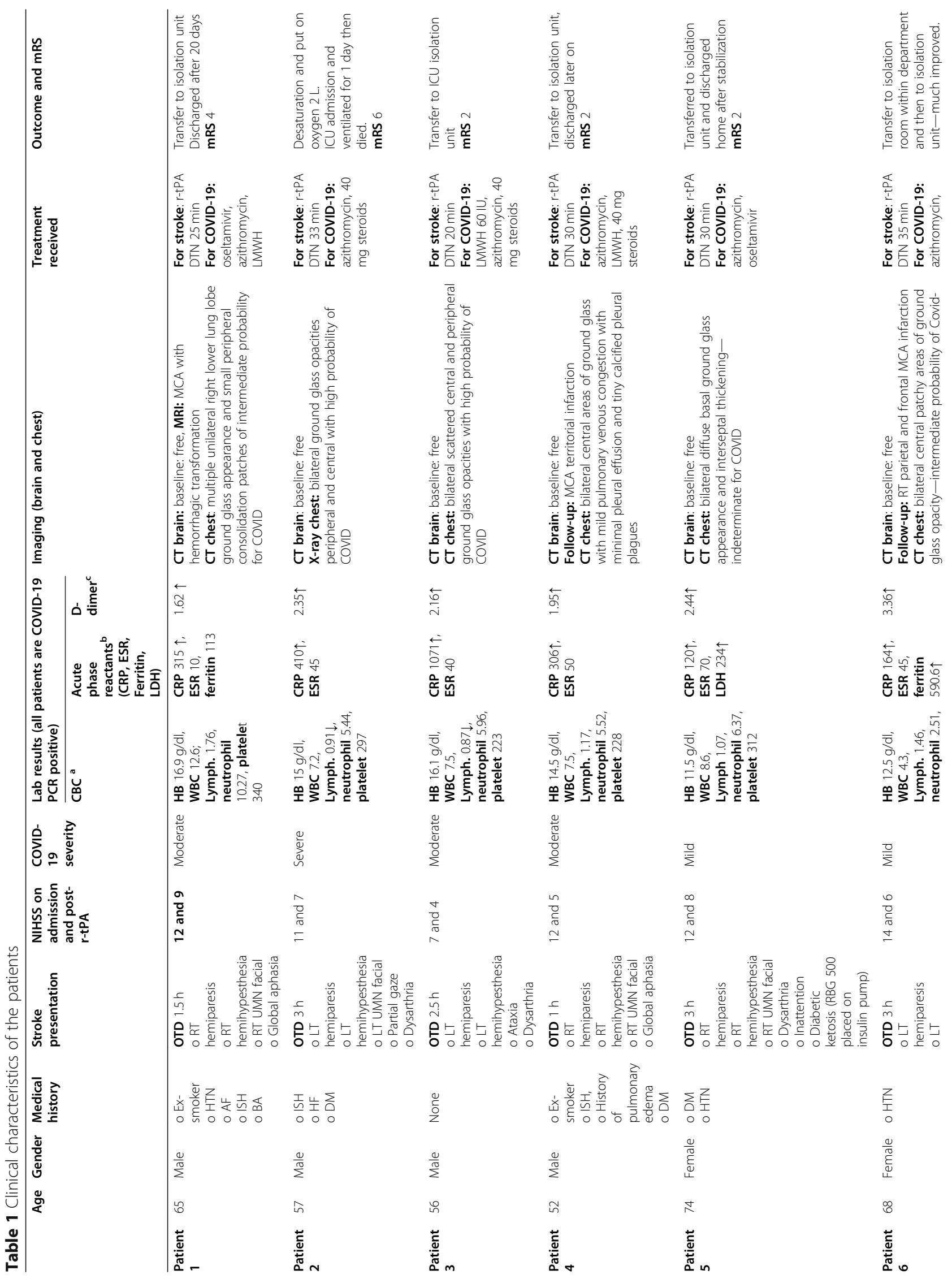




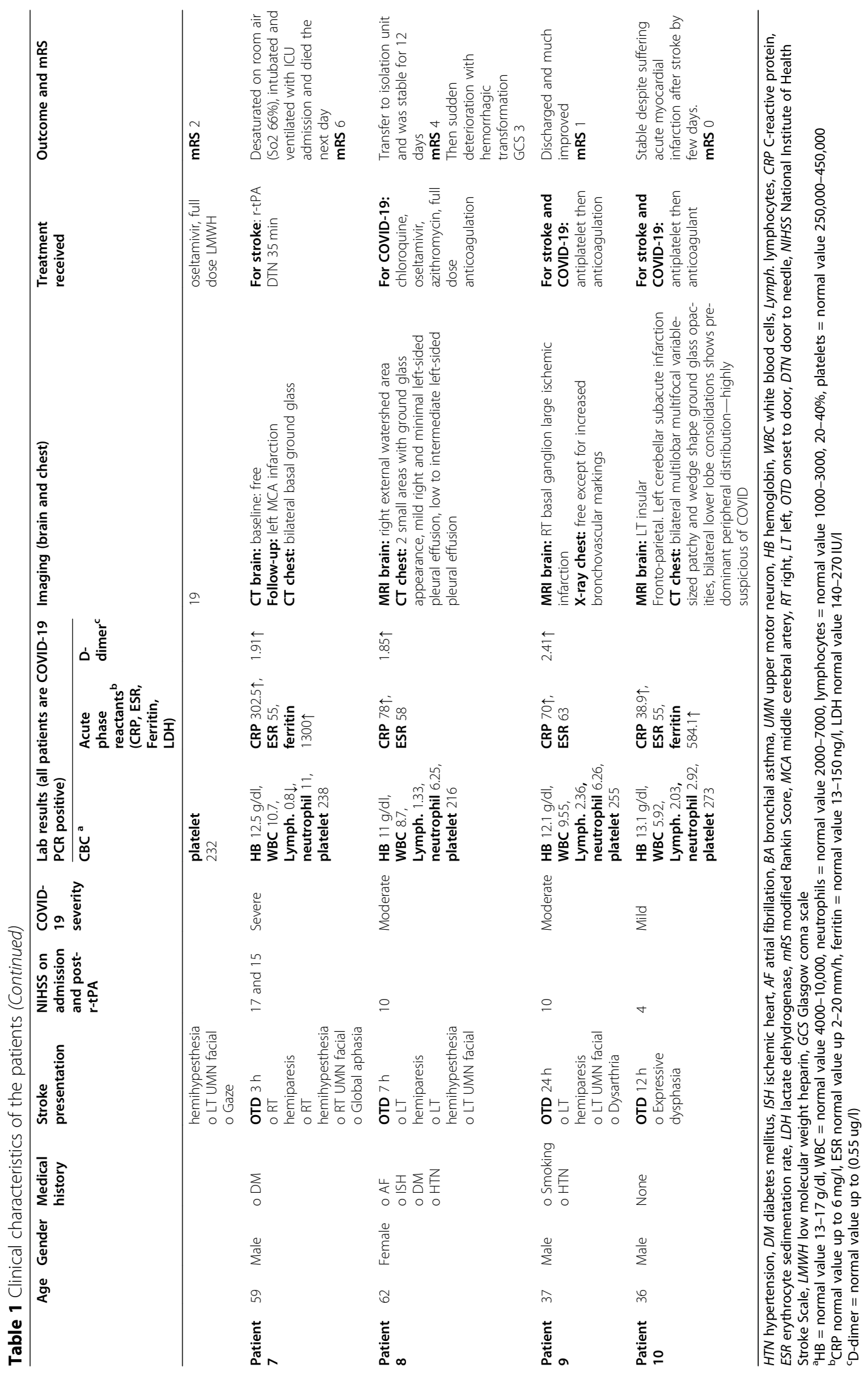


(NIHSS) 12). Investigations showed elevated D-dimer and CRP; computed tomography (CT) chest showed intermediate probability of COVID-19 and normal CT brain. Intravenous thrombolysis (IV r-tPA) was given with door-to-needle time (DNT) of $25 \mathrm{~min}$. Two hours NIHSS was 9, and follow-up magnetic resonance imaging (MRI) (Philips 1.5 Tesla, Germany) showed middle cerebral artery (MCA) M1 territory recent infarction with hemorrhagic transformation. Patient was admitted to isolation unit, then transferred to quarantine hospital and discharged after 20 days with modified Rankin score (mRS) of 4 .

\section{Patient 2}

A 57-year-old male, with IHD, heart failure with EF $37 \%$, and diabetes, presented 4 days following COVID-19 symptoms. ODT was $3 \mathrm{~h}$ of left hemiparesis and partial gaze palsy (NIHSS 11). Investigations showed grade I lymphopenia and elevated D-dimer, CRP, and ESR; Xray chest showed high probability of COVID-19, and CT brain was normal. r-tPA was initiated with DNT of 33 min. Two hours NIHSS was 7. Patient showed signs of desaturation and was mechanically ventilated and died 3 days from admission.

\section{Patient 3}

A 56-year-old male patient, with irrelevant medical history, presented 2 days following COVID-19 symptoms. ODT was 2 and a half hours of left ataxic hemiparesis and dysarthria (NIHSS 7). Investigations showed grade I lymphopenia and elevated D-dimer, CRP, and ESR; CT chest showed high probability of COVID-19 and normal CT brain. r-tPA was given with DNT of $20 \mathrm{~min} ; 2 \mathrm{~h}$ NIHSS was 4. Patient was admitted to isolation ward then to quarantine hospital with mRS 2 .

\section{Patient 4}

A 52-year-old male, ex-smoker, diabetic, IHD, with history of pulmonary edema, EF $38 \%$, presented 3 days following COVID-19 symptoms. ODT was $1 \mathrm{~h}$ of right hemiparesis and global aphasia (NIHSS 12). Investigations showed relative lymphopenia and elevated Ddimer, CRP, and ESR; CT chest showed low probability of COVID, and CT brain was normal. Patient received $\mathrm{r}$ tPA with DNT of $30 \mathrm{~min}$. Two hours NIHSS was 5; follow-up brain imaging showed territorial MCA infarction. Patient was admitted to isolation unit and on obtaining PCR results transferred to quarantine hospital and discharged with mRS 2.

\section{Patient 5}

A 74-year-old female, diabetic and hypertensive, presented 3 days following COVID-19 symptoms. ODT was $3 \mathrm{~h}$ of right hemiparesis and diabetic ketosis for which insulin pump was used (NIHSS 12). Investigations showed relative lymphopenia, elevated D-dimer, and inflammatory markers; CT chest was indeterminate for COVID-1, and CT brain was normal. r-tPA was given with DNT of $30 \mathrm{~min} ; 2 \mathrm{~h}$ NIHSS was 8 . Patient was transferred to isolation unit then to quarantine hospital and discharged home with mRS 2 .

\section{Patient 6}

A 68-year-old female, hypertensive, presented 2 days following COVID-19 symptoms. ODT was $3 \mathrm{~h}$ of left hemiparesis and gaze (NIHSS 14). Investigations showed elevated D-dimer, ferritin, and inflammatory markers; CT chest showed intermediate probability of COVID-19, and CT brain was normal. r-tPA was given with DNT of 35 min; $2 \mathrm{~h}$ NIHSS was 6 . Follow-up MRI showed right MCA territory infarction; patient was transferred to isolation unit then to quarantine hospital with mRS 2 .

\section{Patient 7}

A 59-year-old male, diabetic, presented 2 days following COVID-19 symptoms. ODT was $3 \mathrm{~h}$ of right hemiparesis and global aphasia (NIHSS 17). Investigations showed grade I lymphopenia, elevated D-dimer, ferritin, and inflammatory markers; CT chest showed intermediate probability of COVID-19; CT brain was normal. He received r-tPA with DNT of $35 \mathrm{~min}$; 2 h NIHSS was 15 . MRI brain showed recent left MCA infarction. Patient developed desaturation on room air, $\mathrm{SO}_{2} 66 \%$, intubated and ventilated, and died within 3 days of admission.

\section{Patient 8}

A 62-year-old female, diabetic, hypertensive, with IHD and AF, presented 1 day following COVID-19 symptoms with ODT of $7 \mathrm{~h}$ of left hemiparesis (NIHSS 10). Investigations showed relative lymphopenia, elevated D-dimer and inflammatory markers, and bilateral basal ground glass imaging on CT chest; MRI brain revealed right external watershed infarction. Patient condition was stable over 12 days with mRS 4 , then suddenly deteriorated with Glasgow coma scale 3; CT brain revealed hemorrhagic transformation, interventricular extension, and midline shift (Fig. 1). The patient is still on mechanical ventilator.

\section{Patient 9}

A 37-year-old male, hypertensive and smoker, presented 1 day following COVID-19 symptoms with ODT of 1 day with left hemiparesis and dysarthria (NIHSS 10). Investigations showed elevated $\mathrm{D}$-dimer and inflammatory markers, X-ray chest was normal, and brain imaging revealed right basal ganglionic infarction; patient was admitted to isolation unit with initiation of antiplatelet and 

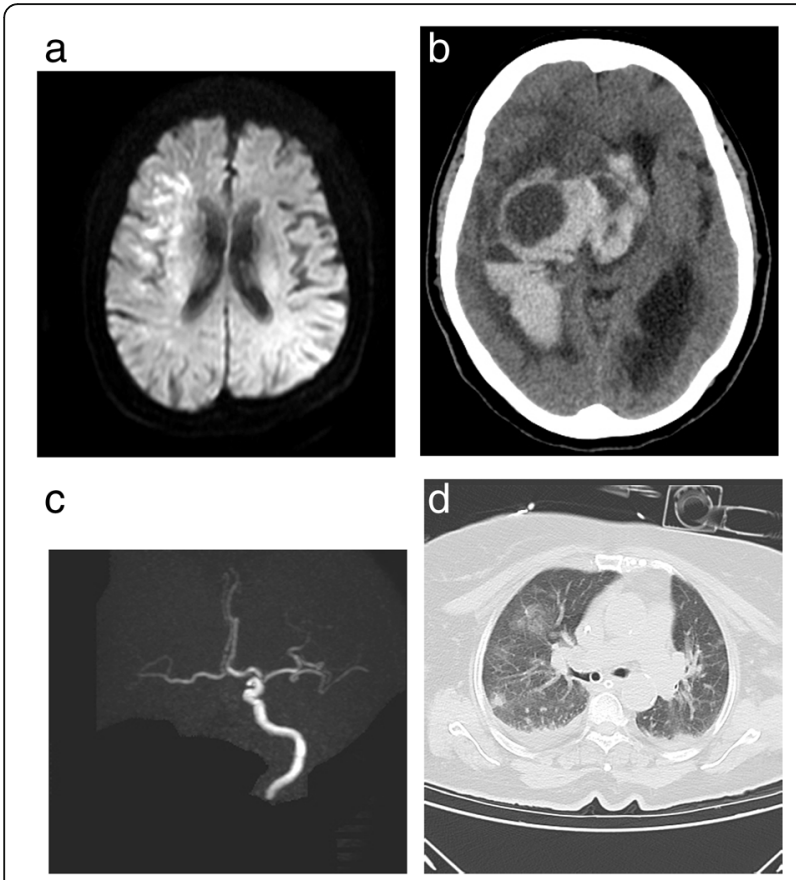

Fig. 1 Patient 8, a MRI DWI with right external watershed infarction, b complete occlusion of right internal carotid artery, c follow-up CT brain after sudden deterioration on day 12 showing hemorrhagic transformation, interventricular extension, and midline shift, d CT chest showed 2 small areas of ground glass appearance, mild right and minimal left-sided pleural effusion, low to intermediate leftsided pleural effusion

anticoagulant, with much improvement and discharged with mRS 1.

\section{Patient 10}

A 36-year-old male, with irrelevant medical history, presented 7 days following COVID-19 symptoms with ODT of $12 \mathrm{~h}$ of aphasia (NIHSS 4). Investigations showed elevated ferritin and inflammatory markers; chest imaging showed high probability of COVID-19; brain imaging showed left insular and left cerebellar subacute infarction. Patient received antiplatelet and was stable with mRS 0, few days later developed acute myocardial infarction; transthoracic echocardiography (Vivid E9 machine, General Electric, Vingmed Ultrasound, Horten, Norway) revealed fresh apical thrombus with elevated troponin $(0.321 \mathrm{ng} / \mathrm{ml}-$ normal range $<0.014 \mathrm{ng} / \mathrm{ml})$, and anticoagulation was initiated (Fig. 2). The patient became stable and was discharged.

\section{Discussion}

All cases presented with AIS ranging from 1 to 7 days after onset of viremia symptoms which included fever, dyspnea, fatigue, and/or myalgia. CT chest was suggestive of COVID-19 criteria; D-dimer was mildly elevated, with moderately to markedly increased CRP and ESR. Lymphocytic count ranged from normal to relative or grade I lymphopenia. Similar findings were reported by other groups [5-7]. All cases were confirmed with a positive PCR within $48 \mathrm{~h}$ of presentation.

Seven patients received thrombolytic therapy, and the rest either anticoagulant or antiplatelet therapy. Six patients improved as regards stroke with mRS ranging from 0 to 4 .

Four out of ten patients (patients 2, 7, 8, and 10) developed complications. Only patient 8 had a neurological cause for deterioration, while patients 2 and 7 had sudden desaturation and eventually died. Patient 10 reached $\mathrm{mRS}=0$, then had a myocardial infarction, but fortunately, he recovered and was discharged. This agrees with what has been suggested that AIS might be associated with severe form of COVID-19 [8].

The controversy whether COVID-19 can cause thrombosis or hemorrhage still holds, as seen in patient 8 who developed hemorrhagic transformation of her infarction. Although patients with increased D-dimer can suffer from thrombotic events, yet hemorrhage due to prolonged prothrombin time might occur [6].

It is worth mentioning that cases 3 and 10 had no risk factors for stroke; in addition, case 10 was only 36 years old yet developed myocardial infarction few days after AIS. This raises the possibility of occurrence of an inflammatory response termed "immunothrombosis" in such cases [9-11]. However, still one cannot overlook the detrimental effect of hyperthermia, dehydration, and hypoxia in the presence of viral endothelial invasion through ACE receptors that are abundant in endothelial cells $[5,12,13]$. It is also reported that COVID-19 has a role in developing diffuse vasculopathy and vasculitis through inflammatory cell infiltration, endothelial cell damage, and endotheliopathy. All these factors can contribute to platelet aggregation and thrombosis [5].

Out of the current case series, we have the following recommendations: COVID-19 has pulmonary besides extrapulmonary manifestations, and stroke is one of such manifestations, so triaging patients in ER with a proper baseline investigations as CT chest and nasopharyngeal swab can detect hidden COVID-19 cases that ought to be isolated and at the same time managed as stroke guidelines recommend based on onset to door. In our presented cases D-dimer was mildly elevated and lymphocytic count was showing grade I lymphopenia in some but not all cases so it is worth mentioning that other supportive criteria for COVID-19 like symptomatology, CT chest, and PCR are of greater value than labs. 

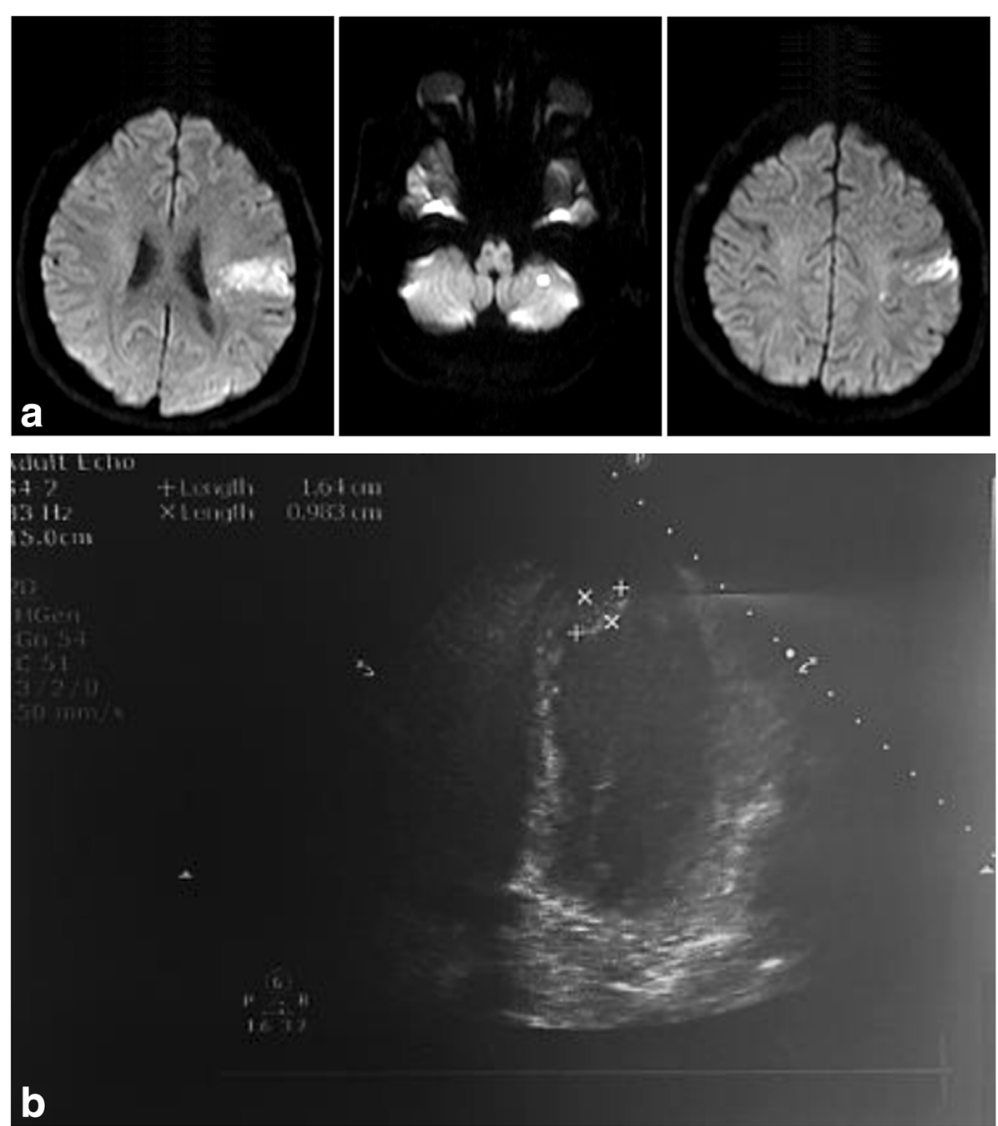

Fig. 2 a MRI DWI of patient 10 with left insular and cerebellar infarction. b ECHO of patient 10 with fresh apical thrombus measuring $12 \times 16$ mm, EF 35\%, akinetic all apical segment, mid-anterior wall, mid-anterior septum, and mid-posterior septum

\section{Conclusion}

Whether a cause or a coincidence, COVID-19 with stroke has been treated by r-tPA, anticoagulants, and/or antiplatelets. Patient 8 developed intracerebral hemorrhage after receiving anticoagulation, while case 10 developed another thrombotic event despite receiving anticoagulant and antiplatelet. So in order to achieve the best outcome and avoid complications, guidelines for the timing and dosing of medications that guard against immunothrombosis need further elaboration.

\section{Abbreviations}

COVID-19: Coronavirus disease 2019; AIS: Acute ischemic stroke;

PCR: Polymerase chain reaction; IHD: Ischemic heart disease; AF: Atrial fibrillation; ODT: Onset-to-door time; MRI: Magnetic resonance imaging; MCA: Middle cerebral artery; NIHSS: National Institutes of Health Stroke Scale; CT: Computed tomography; IV r-tPA: Intravenous thrombolysis; DNT: Door-toneedle time; mRS: Modified Rankin score

\section{Acknowledgements}

Not applicable.

\section{Authors' contributions}

NE: design and conceptualized the study. TR: conception of the work and drafting the manuscript. EH: acquisition and analysis of data. SF: acquisition and analysis of data. HS: conception of the work and drafting the manuscript. MF: acquisition and analysis of data. EA: data collection and manuscript revision. $\mathrm{AH}$ : contributor in writing the manuscript. $\mathrm{HA}$ : design and conceptualized the study. All authors have agreed to conditions noted on the Authorship Agreement Form and have read and approved the final version submitted. The content of the manuscript has not been published or submitted for publication elsewhere. The author(s) read and approved the final manuscript.

\section{Funding}

No funds were received to fulfill this work.

\section{Availability of data and materials}

The corresponding author takes full responsibility for the data, has full access to all of the data, and has the right to publish any and all data separate and apart from any sponsor.

\section{Ethics approval and consent to participate}

All procedures performed in the study were in accordance with the ethical standards of the Faculty of Medicine, Ain Shams University Research and Ethical Committee, and with the 1964 Helsinki declaration and its later amendments or comparable ethical standards. We obtained approval from research ethics committee no. FWA 000017585. On 27 August 2020.

\section{Consent for publication}

Written informed consent was obtained from participants or next of kin for publication of this case series and accompanying images. We obtained approval from research ethics committee no. FWA 000017585. On 27 August 2020.

\section{Competing interests}

None of the authors has any conflict of interest. 
Received: 9 October 2020 Accepted: 9 December 2020

Published online: 24 December 2020

References

1. Huang C, Wang Y, Li X, Ren L, Zhao J, Hu Y, et al. Clinical features of patients infected with 2019 novel coronavirus in Wuhan, China. Lancet. 2020:395(10223):497-506

2. Wang H-Y, Li X-L, Yan Z-R, Sun X-P, Han J, Zhang B-W. Potential neurological symptoms of COVID-19. Ther Adv Neurol Disord. 2020;13:1-2.

3. Mao L, Jin H, Wang M, Hu Y, Chen S, He Q, et al. Neurologic manifestations of hospitalized patients with coronavirus disease 2019 in Wuhan, China. JAMA Neurol. 2020;77(6):683-90.

4. Roushdy TM, Nahas NME, Aref HM, et al. Stroke in the time of coronavirus disease 2019: experience of two university stroke centers in Egypt. J Stroke. 2020:22(2):275-7. https://doi.org/10.5853/jos.2020.01550.

5. Tang N, Li D, Wang X, Sun Z. Abnormal coagulation parameters are associated with poor prognosis in patients with novel coronavirus pneumonia. J Thromb Haemost. 2020;18(4):844-7.

6. Wang D, Hu B, Hu C, Zhu F, Liu X, Zhang J, et al. Clinical characteristics of 138 hospitalized patients with 2019 novel coronavirus-infected pneumonia in Wuhan, China. JAMA. 2020;323(11):1061-9.

7. Zhou F, Yu T, Du R, Fan G, Liu Y, Liu Z, et al. Clinical course and risk factors for mortality of adult inpatients with COVID-19 in Wuhan, China: a retrospective cohort study. Lancet. 2020;395(10229):1054-62.

8. Aggarwal G, Lippi G, Michael HB. Cerebrovascular disease is associated with an increased disease severity in patients with coronavirus disease 2019 (COVID-19): a pooled analysis of published literature. Int. J. Stroke. 2020; 15(4):385-9.

9. Connors JM, Levy JH. COVID-19 and its implications for thrombosis and anticoagulation. Blood. 2020;135(23):2033-40.

10. Engelmann B, Massberg S. Thrombosis as an intravascular effector of innate immunity. Nat Rev Immunol. 2013;13(1):34-45.

11. Jackson SP, Darbousset R, Schoenwaelder SM. Thromboinflammation: challenges of therapeutically targeting coagulation and other host defense mechanisms. Blood. 2019:133(9):906-18.

12. Fan B, Chong V, Chan S, Lim G, Guan K, Lim E, et al. Hematologic parameters in patients with COVID-19 infection. Am J Hematol. 2020;95(6): $131-4$.

13. Riphagen S, Gomez R, Gonzalez-Martinez C, Wilkinson N, Theocharis P. Hyperinflammatory shock in children during COVID-19 pandemic. Lancet. 2020;395(10237):1607-8.

\section{Publisher's Note}

Springer Nature remains neutral with regard to jurisdictional claims in published maps and institutional affiliations.

\section{Submit your manuscript to a SpringerOpen ${ }^{\circ}$ journal and benefit from:}

- Convenient online submission

- Rigorous peer review

- Open access: articles freely available online

High visibility within the field

- Retaining the copyright to your article

Submit your next manuscript at $\boldsymbol{\nabla}$ springeropen.com 